\title{
The role of attention-deficit/hyperactivity disorder in the association between verbal ability and conduct disorder
}

\author{
Ashley K. Smith ${ }^{1,2}$, Shannon M. Stasi', Soo Hyun Rhee ${ }^{1,2}{ }^{*}$, Robin P. Corley $^{2}$, Susan E. Young ${ }^{2}$ \\ and John K. Hewitt ${ }^{1,2}$
}

\author{
1 Department of Psychology and Neurosciences, University of Colorado, Boulder, CO, USA \\ 2 Institute for Behavioral Genetics, University of Colorado, Boulder, CO, USA
}

Edited by:

Nancy Zucker, Duke University Medical Center, USA

Reviewed by:

Jason Decaro, University of Alabama, USA

\section{*Correspondence:}

Soo Hyun Rhee, Department of Psychology and Neuroscience, University of Colorado, 345 UCB, Boulder, CO 80309, USA.

e-mail:soo.rhee@colorado.edu
Although there is clear evidence that low verbal ability is a risk factor for conduct disorder (CD), some researchers have questioned whether this association is due to the common comorbidity between attention-deficit/hyperactivity disorder (ADHD) and CD. The present study examined the association among verbal ability, ADHD, and $C D$ in a genetically informative sample in order to examine the role of genes and/or environmental influences shared in common with ADHD on the covariation between verbal ability and CD. Participants were 2744 adolescents from the Center for Antisocial Drug Dependence (CADD), and included 360 monozygotic (MZ) female twin pairs, 221 dizygotic (DZ) female twin pairs, 297 MZ male twin pairs, 220 DZ male twin pairs, and 274 opposite-sex DZ twin pairs. The Diagnostic Interview Schedule for Children (DISC-IV) was used to assess lifetime symptoms of ADHD and CD. Verbal ability was assessed via the Vocabulary subtest of theWechsler Adult Intelligence Scale III (WAIS-III) for individuals over the age of 16 and the Vocabulary subtest of the Wechsler Intelligence Scale for Children III (WISCIII) for individuals under the age of 16 . There was a small but significant negative covariance between verbal ability and $C D$ and between verbal ability and ADHD. Results also suggest that the covariation between verbal ability and $C D$ is due to influences shared in common with ADHD.

Keywords: conduct disorder, verbal ability, attention-deficit/hyperactivity disorder, comorbidity, behavioral genetics

\section{INTRODUCTION}

Conduct disorder (CD) is defined by the Diagnostic and Statistical Manual of Mental Disorders as a repetitive and persistent pattern of behavior in which the basic rights of others or major age-appropriate societal norms or rules are violated (American Psychiatric Association, 2000). CD is one of the most common psychiatric disorders in childhood, with prevalence ranging from 6 to $16 \%$ for males and from 2 to $9 \%$ for females (American Psychiatric Association, 2000). Further, it is evident that $\mathrm{CD}$ has negative consequences not only for the child but those in their life and society. Children with CD often experience poor academic achievement, low employment and socioeconomic status, higher rates of automobile accidents, family problems, antisocial behavior, and mood problems in adulthood (e.g., Waschbusch, 2002). Therefore, gaining a greater understanding of $\mathrm{CD}$ and its etiology is important.

Several lines of evidence suggest that low verbal ability is a risk factor for CD (e.g., Wechsler, 1944; Moffitt and Silva, 1988a). A review paper reports that delinquent individuals have average full scale or verbal intelligence quotient (IQ) scores 8-10 points lower than the population average (Nigg and Huang-Pollock, 2003). Quay's (1987) review also found that delinquent adolescents have lower IQ scores than adolescents from general population samples, and that the delinquents' deficits were stronger in the verbal domain. Approximately two-thirds of delinquents were deficient in verbal abilities measured by standardized intelligence tests. Longitudinal studies also find that early weakness in verbal learning and verbal reasoning ability predicts later persistent offending, CD, and antisocial outcomes (e.g., Moffitt, 1990a; Farrington and
Hawkins, 1991). The verbal deficit in youth with CD appears to be at least partially independent of such potential confounds as ethnicity and socioeconomic status (e.g., Hirschi and Hindelang, 1977; Wilson and Herrnstein, 1985; Petee and Walsh, 1987; Lynam et al., 1993; Moffitt et al., 1994).

Researchers have proposed several theories explaining the association between VA and CD (see Moffitt, 1990b for a review). Luria (1963) posited that because speech processes are less developed in children with verbal deficits, they have a difficult time evaluating novel situations while simultaneously incorporating past and present information, leading to difficulties in complying with behavioral rules. Savitsky and Czyzewski (1978) suggested that verbal deficits may lead to mislabeling the emotions of others, which could lead to improper reactions. Also, verbal deficiencies may hinder a person's ability to anticipate consequences of behaviors as well as to decide what is morally right and wrong (Wilson and Herrnstein, 1985). Further, Loney et al. (1998) suggested that verbal deficits affect engagement in private speech, which is an important factor in behavior regulation. School failure may play a role in the link between verbal deficits and delinquency, as it may result in social rejection, which then decreases the chances one will identify and socialize with prosocial peers (Moffitt, 1993).

Although it is clear that low verbal ability is a risk factor for CD, several researchers have questioned whether this association is due to the comorbidity between attention-deficit/hyperactivity disorder (ADHD) and CD. ADHD, which is characterized by symptoms of inattention, impulsivity, and/or hyperactivity, is significantly comorbid with $\mathrm{CD}$; for example, one meta-analysis reported a 
median odds ratio of 10.7 for comorbidity between ADHD and CD (Angold et al., 1999). A review by Hogan (1999) notes 16 of 27 studies examining the association between $\mathrm{CD}$ and general intelligence measures failed to control for ADHD symptoms, and that studies that did control for ADHD typically found no or diminished association between CD and IQ.

Henry and Moffitt (1997) noted that verbal deficits are especially pronounced in delinquents with ADHD. Also, Moffitt and Silva (1988b) noted that although a group with only histories of delinquency had significantly lower verbal scores than a comparison group, the delinquency plus attention-deficit disorder group was significantly more impaired than the delinquency only group. A meta-analysis conducted by Waschbusch (2002) reported differences in verbal ability among children with hyperactive-impulsive attention (HIA) problems and conduct problems. Although children with both HIA and conduct problems and children with HIA only had significantly lower verbal IQ scores than comparison group, the conduct problem-only group did not differ from controls.

In contrast, Déry et al. (1999) found results that did not support the hypothesis that the lower verbal ability in $\mathrm{CD}$ adolescents is due to comorbidity with ADHD. Both CD + ADHD and CD-only groups had significantly lower verbal scores than the comparison group. Although the CD + ADHD group had lower verbal scores than the CD-only group, the difference was not statistically significant, suggesting that lower verbal scores are associated with $\mathrm{CD}$, not with comorbid CD + ADHD.

Given the possible role of ADHD in the association between $\mathrm{CD}$ and verbal ability, we conducted a genetically informative study examining whether $\mathrm{CD}$ is influenced by genetic and/or environmental influences shared in common with both ADHD and verbal ability and those shared in common only with verbal ability (after controlling for the ADHD). Twin studies estimate the magnitude of genetic and environmental influences on phenotypes such as $\mathrm{CD}$ and verbal ability by comparing the similarity between monozygotic (MZ) twin pairs and dizygotic (DZ) twin pairs. MZ twins share $100 \%$ of their genes, whereas DZ twins and ordinary siblings share $50 \%$ of their genes identical by descent. Thus, there is evidence of genetic influences on a trait if MZ twins are more similar than DZ twins or ordinary siblings on phenotypic traits. The proportion of observed variance in the population that can be explained by genetic differences is heritability $\left(\mathrm{A}\right.$ or $\left.\mathrm{a}^{2}\right)$. Twin studies also differentiate between shared environmental $\left(\mathrm{C}\right.$ or $\left.\mathrm{c}^{2}\right)$, influences, which make individuals in a family more similar and non-shared environmental influences $\left(\mathrm{E}_{\mathrm{for}} \mathrm{e}^{2}\right)$, which make individuals in a family dissimilar on a particular trait.

Twin studies also can quantify the magnitude of genetic, shared environmental, and non-shared environmental influences on the covariance between two traits, such as CD and verbal ability. In bivariate genetic analyses, cross-sibling, cross-trait correlations (e.g., the correlation between $\mathrm{CD}$ in twin 1 and verbal ability in twin 2 or vice versa) in $M Z$ and $D Z$ twin pairs are compared. There is evidence of genetic influence on the covariance between two traits if the MZ cross-sibling, cross-trait correlation is greater than that of DZ twins or of full siblings.

To our knowledge, there has not been a previous behavior genetic study examining the associations among $\mathrm{CD}$, verbal ability, and ADHD. However, there is a large literature examining the genetics of $\mathrm{CD}, \mathrm{ADHD}$, and verbal ability. A meta-analysis by Rhee and Waldman (2002) concluded that there were significant and shared environmental influences on $\mathrm{CD}\left(\mathrm{a}^{2}\right.$ of $0.50, \mathrm{c}^{2}$ of 0.11 , and an $\mathrm{e}^{2}$ of 0.39 ). Additionally, a review of genetics studies examining verbal ability indicates an overall heritability of $65 \%$ (Plomin and Craig, 1997). A review of 20 twin studies of ADHD reported a mean heritability estimate of $76 \%$ and little evidence of shared environmental influences on ADHD (Faraone et al., 2005). Also, several studies have suggested that there are common genetic influences on ADHD and CD (e.g., Thapar et al., 2001; Nadder et al., 2002; Dick et al., 2005). There is little known regarding the effects of genes and environmental influences on the covariation between verbal ability and ADHD/CD. Plomin et al. (2002) examined the association between verbal and non-verbal cognitive abilities and behavior problems in preschool children, and found evidence of both genetic and shared environmental influences on the covariance between cognitive ability and behavior problems. Koenen et al. (2006) found significant genetic influences on the association between general IQ and antisocial behavior in males, but not females, across the ages of 5-7. When individuals with ADHD were excluded from the analyses, the correlations were slightly attenuated, but the associations were still significant for males.

In sum, previous studies suggest that the association between verbal ability deficits and $\mathrm{CD}$ may be due to the comorbidity between ADHD and CD. Given this suggestion, we investigated the etiology of $\mathrm{CD}$, examining whether low verbal ability is a significant independent risk factor for $\mathrm{CD}$ after controlling for ADHD. The first alternative hypothesis is that there are unique genetic and environmental influences shared between verbal ability and CD, independent from those shared with ADHD. The second alternative hypothesis is that the common influences shared between verbal ability and $\mathrm{CD}$ are also shared with $\mathrm{ADHD}$. To investigate these alternative hypotheses, the etiology of $\mathrm{CD}$ was examined by decomposing its variance into influences shared with both verbal ability and ADHD, those shared only with verbal ability, and $\mathrm{CD}$-specific variance, in a genetically informative sample. Per alternative hypothesis 1 , if there are significant genetic and/or environmental influences shared only with verbal ability (after accounting for ADHD) on CD, this would be evidence for verbal ability as an independent risk factor for CD. Per alternative hypothesis 2 , if there are not significant shared genetic and environmental influences between verbal ability and CD after controlling for ADHD, this would suggest that low verbal ability is not an independent risk factor for $\mathrm{CD}$. The trivariate Cholesky decomposition model (see Analyses for a detailed explanation) was used to address this question.

\section{MATERIALS AND METHODS \\ PARTICIPANTS}

The present study includes 2744 adolescents from the Center for Antisocial Drug Dependence (CADD), an ongoing, multi-component, collaborative study underway at the Institute for Behavioral Genetics (IBG) at the University of Colorado. The age range of the sample was 11.33-19.01 years $(M=14.99, \mathrm{SD}=2.16)$. Among these participants, there were $360 \mathrm{MZ}$ female twin pairs, $221 \mathrm{DZ}$ female twin pairs, $297 \mathrm{MZ}$ male twin pairs, $220 \mathrm{DZ}$ male twin pairs, and 274 opposite-sex DZ twin pairs recruited from two community-based 
samples: the Colorado longitudinal twin sample (LTS) and community twin sample (CTS). The LTS and CTS are two samples recruited into the CADD from the Colorado twin registry (CTR), a population-based registry housed at IBG at the University of Colorado. The LTS consists of a sample of twins whose development has been studied since birth, and the CTS is a sample of twins recruited for the first time by the CADD. Data for the present study were collected from the two samples over the same period of time. Informed consent and assent forms approved by the University of Colorado Human Research Committee were completed by participants and their guardians before the start of study procedures. Further information concerning the recruitment and sample description of both samples can be found in Rhea et al. (2006).

\section{PROCEDURES}

To determine the zygosity of the same-sex twin pairs, a nine-item assessment of physical characteristics was completed by interviewers. This assessment was compared to concordance of the twin pairs' genotype at 11 highly informative short tandem repeat polymorphisms. Twin pairs with similar physical characteristics and concordant genotypic markers were classified as MZ pairs. Those with disparate physical features and genotypes were classified as DZ pairs. Among these, only nine had discordant physical characteristic assessments and DNA data. These discordances were re-examined and resolved by inspection of photographs of facial structure and ear morphology, and DNA recollection and regenotyping if necessary.

In the present study, the Diagnostic Interview Schedule for Children - IV (DISC-IV; Shaffer et al., 2000) was used to assess ADHD and CD. The DISC-IV is a structured psychiatric interview assessing DSM-IV (American Psychiatric Association, 1994) symptoms and diagnoses for Axis I disorders over the past year as well as lifetime, based on adolescent interviews. An earlier study utilizing a subset of the same sample (Ehringer et al., 2006) demonstrated that the prevalence rates for $\mathrm{ADHD}$ and $\mathrm{CD}$ are comparable to those reported in the Methods for the Epidemiology of Child and Adolescent Mental Disorders Study (Shaffer et al., 1996). To determine the absence or presence of symptoms, and whether diagnostic thresholds were met, computer algorithms were developed in accordance with instructions from the instrument's authors. Because of the low prevalence of ADHD and CD in this population-based sample, only the symptom criteria were used, and DSM-IV criteria for age of onset of symptoms or symptom clustering were not used. Therefore, caution should be used when interpreting prevalence rates for $\mathrm{CD}$ and $\mathrm{ADHD}$ diagnoses in our sample (see Table 1). Verbal ability was assessed via the vocabulary subtest of the Wechsler adult intelligence scale III (WAIS-III; Wechsler, 1997a) for individuals 16 years of age and older or via the Vocabulary subtest of the Wechsler intelligence scale for children III (WISC-III; Wechsler, 1991) for individuals under the age of 16 . The verbal scores are the standardized residuals after the effects of age and sex have been regressed out and have a mean of zero and variance of 1.0.

\section{ANALYSES}

To investigate the covariation among ADHD, CD, and verbal ability, multivariate genetic analyses were conducted using Mplus (Muthén and Muthén, 1998-2004). Mplus allows analyses of a combination of continuous and ordinal variables, which is ideal for the present study, given that verbal ability is a continuous variable, and ADHD and CD are ordinal variables. Because DSM-IV psychiatric symptoms are highly skewed, data were analyzed with the assumption that a normal continuous liability distribution underlies the ordinal variables (i.e., $0=$ no symptoms, $1=$ one or more symptoms, and 2 = diagnosis). This approach allows for statistical analyses that preserve the statistical advantages of the normality assumptions for the underlying liability, retains an explicit mapping between the underlying liability and observed data, and provides accurate measures of the underlying correlations and parameter estimates (Stallings et al., 2001; Derks et al., 2004). Also, given significant sex and age differences in the prevalence of symptoms and diagnosis of ADHD and CD, sex-specific thresholds were estimated, and age was included as a covariate in the genetic analyses. Effects of age and sex were regressed out from verbal ability prior to analyses.

\section{Correlations}

Phenotypic correlations among ADHD, CD, and verbal ability were estimated, with polychoric correlations being calculated between the two ordinal variables (ADHD and CD) and polyserial correlations

Table 1 | Number of individuals (and percentages of sample) with no CD/ADHD symptoms, subthreshold CD/ADHD symptoms, and diagnosis of CD/ADHD.

\begin{tabular}{|c|c|c|c|c|c|c|}
\hline & \multirow[b]{2}{*}{ Entire sample } & \multicolumn{2}{|c|}{ MZ } & \multicolumn{3}{|c|}{ DZ } \\
\hline & & Male & Female & Male & Female & os \\
\hline Absent & 1483(54.0) & $286(48.1)$ & $433(60.1)$ & $207(47.0)$ & $294(66.5)$ & $263(48.0)$ \\
\hline Subthreshold & 1027(37.4) & $228(38.4)$ & $254(35.3)$ & 186(42.3) & $128(29.0)$ & $231(42.2)$ \\
\hline Diagnosis & 234(8.5) & $80(13.5)$ & $33(4.6)$ & $47(10.7)$ & $20(4.5)$ & 54(9.9) \\
\hline \multicolumn{7}{|l|}{ ADHD } \\
\hline Absent & 1596(58.2) & $345(58.1)$ & $427(59.3)$ & 237(53.9) & $276(62.4)$ & $311(56.8)$ \\
\hline Subthreshold & $1051(38.3)$ & 229(38.6) & $276(38.3)$ & $181(41.1)$ & $152(34.4)$ & 213(38.9) \\
\hline Diagnosis & 97(3.5) & 20(3.4) & $17(2.4)$ & $22(5.0)$ & 14(3.2) & $24(4.4)$ \\
\hline Total & $2744(100)$ & $594(100)$ & $720(100)$ & $440(100)$ & $442(100)$ & $548(100)$ \\
\hline
\end{tabular}


being calculated between the continuous variable (verbal scores) and the two ordinal variables (ADHD and CD). Within-trait crosssibling correlations and cross-trait cross-sibling correlations were estimated for $\mathrm{MZ}$ and $\mathrm{DZ}$ twin pairs.

\section{Multivariate model}

Figure 1 presents the Cholesky decomposition model, which was tested to examine the role of ADHD in the association between CD and verbal ability and the degree to which this covariation is due to genetic, shared environmental, or non-shared environmental influences. In the Cholesky model, the number of genetic, shared environmental, and non-shared environmental factors are equal to the number of variables entered in the model. The first set of factors influences ADHD, verbal ability, and CD; the second set of factors influences only verbal ability and CD; the third set of factors influences only CD. In this model, the first variable is ADHD, the second variable is verbal ability, and the third variable is CD. The variance of CD is divided into that shared in common with both

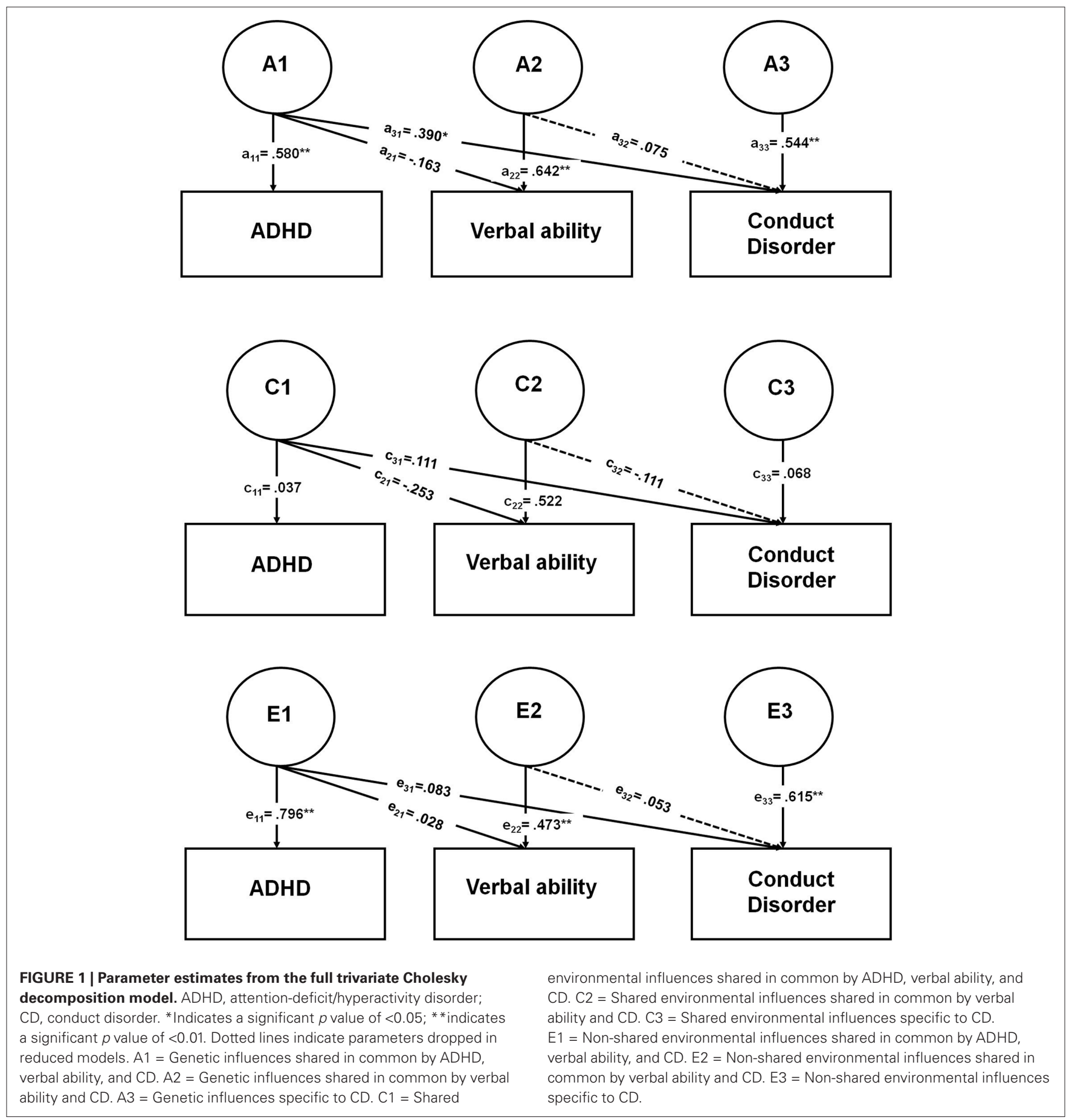


ADHD and verbal ability, that shared with verbal ability alone, and $\mathrm{CD}$-specific variance. The path coefficient $a_{11}$ is the effect of A1 on the first variable, ADHD; the path coefficient $a_{21}$ is the effect of A1 on the second variable, verbal ability; the path coefficient $a_{31}$ is the effect of $\mathrm{A} 1$ on the third variable, $\mathrm{CD}$, and so on. The interpretation of $\mathrm{C} 1, \mathrm{C} 2, \mathrm{C} 3, \mathrm{E} 1, \mathrm{E} 2$, and E3 and the corresponding path coefficients are similar, except $\mathrm{C}$ refers to shared environmental influences, and E refers to non-shared environmental influences. To test the significance of the genetic, shared environmental, and non-shared environmental influences on CD that are shared in common with verbal ability alone (after controlling for the influences of ADHD), paths $a_{32}, c_{32}$, and $e_{32}$ were dropped (see dotted lines in Figure 1), using the chi-square difference test.

Additionally, because several studies suggest that the heritability of intelligence changes over the life course (e.g., Plomin and Spinath, 2004; Hoekstra et al., 2007), we examined whether the results are significantly different for younger and older participants. A model where parameter estimates were free to vary across younger (11-14.99) and older participants (15-19) was compared to one where they were constrained to be equal across younger and older participants.

\section{RESULTS}

\section{PRELIMINARY ANALYSES}

A model allowing correlations to be different between males and females $\left[\chi^{2}(39)=39.37, p=0.45 ; \mathrm{CFI}=1.00\right.$; $\left.\mathrm{RMSEA}=0.006\right]$ and a model where male and female correlations were constrained to be the same $\left[\chi^{2}(60)=67.87, p=0.23\right.$; CFI $=0.99$; $\left.\mathrm{RMSEA}=0.022\right]$ were tested. The fit of these two models was not significantly different $\left[\Delta \chi^{2}(21)=28.87, p=0.12\right]$, suggesting that the male and female correlations were not significantly different. Table 2 presents the phenotypic correlations among ADHD, CD, and verbal ability. Phenotypic correlations between ADHD and CD were positive and statistically significant, such that individuals with ADHD were also more likely to have CD. Phenotypic correlations between verbal ability and ADHD and between verbal ability and CD were negative and statistically significant, indicating that lower verbal ability is associated with increased likelihood of ADHD and CD.

Table 3 presents the within-trait cross-twin correlations and the cross-trait cross-twin correlations in MZ and DZ twin pairs. Correlations are provided for descriptive purposes. The model fitting results (in the Primary Analyses) provide a statistical test of the following observations. Among the within-trait cross-twin correlations, $\mathrm{MZ}$ correlations were greater than $\mathrm{DZ}$ correlations for $\mathrm{ADHD}$, verbal ability, and CD for both sexes, suggesting genetic influences on

Table 2 | Phenotypic correlations among disorders.

\begin{tabular}{|c|c|c|c|c|c|c|c|c|}
\hline & \multirow[b]{2}{*}{ ADHD } & \multicolumn{2}{|c|}{ Full sample } & \multicolumn{2}{|c|}{ Males } & \multicolumn{3}{|c|}{ Females } \\
\hline & & Verbal & CD & ADHD & Verbal CD & ADHD & Verbal & CD \\
\hline \multicolumn{9}{|c|}{ ADHD } \\
\hline \multicolumn{3}{|c|}{ Verbal $-0.081 * *$} & & \multicolumn{2}{|c|}{$-0.089 * *$} & \multicolumn{3}{|c|}{$-0.072^{*}$} \\
\hline CD & $0.356 * *$ & $-0.073^{* *}$ & & $0.367^{* *}$ & -0.033 & $0.343^{* *}$ & $-0.112^{* *}$ & \\
\hline
\end{tabular}

*Indicates a significant $p$ value of $<0.05 ; *{ }^{*}$ indicates a significant $p$ value of $<0.01$. all variables. The cross-trait cross-twin correlations showed greater MZ correlations than DZ correlations for the covariance between ADHD and verbal ability and between ADHD and CD, but not between $\mathrm{CD}$ and verbal ability. This finding suggests genetic influences on the covariance between ADHD and verbal ability and ADHD and $\mathrm{CD}$, but not on the covariance between $\mathrm{CD}$ and verbal ability.

\section{PRIMARY ANALYSES}

To examine the statistical significance of genetic and environmental influences on ADHD, verbal ability, CD, model fitting analyses were conducted. The full Cholesky decomposition model fit the data adequately $\left[\chi^{2}(152)=212.80, p<0.01, \mathrm{CFI}=0.95\right.$, TLI $=0.97$, RMSEA $=0.04$; Hu and Bentler (1998) suggested that a CFI and TLI greater than 0.95 and RMSEA less than 0.06 indicate good model fit]. Dropping the influences shared only between verbal ability and CD (i.e., dropping the paths $a_{32}, c_{32}$, and $e_{32}$ simultaneously from the model shown in Figure 1) from the Cholesky model from the full model (using the DIFFTEST option in Mplus for WLSMV estimation) did not lead to a significant decrement in the fit of the model $\left[\Delta \chi^{2}(3)=2.40, p=0.49\right]$. The reduced model also fit the data adequately $\left[\chi^{2}(155)=215.44, p<0.01, \mathrm{CFI}=0.95\right.$, $\mathrm{TLI}=0.97$, RMSEA $=0.04]$. We tested whether the parameters were significantly different in two age groups: $11-14.99$ and 15-19. Constraining the parameters to be equal across younger and older groups did not lead to a significant decrement in the fit of the model $\left[\Delta \chi^{2}(17)=17.82, p=0.40\right]$.

Figure 1 shows the parameter estimates from the full trivariate Cholesky decomposition model (statistically significant parameter estimates are indicated with an asterisk), in which the variance of CD was decomposed into variance shared in common with both ADHD and verbal ability, variance shared in common with verbal ability only, and variance unique to $\mathrm{CD}$. The parameter estimates from Figure 1 were squared to calculate the percentage of variance of ADHD, verbal ability, and CD explained by the three sets of genetic, shared environmental, and non-shared environmental influences (i.e., A1, C1, E1, A2, C2, E2, A3, C3, and E3) and the variance explained by age (for the two ordinal variables, ADHD and CD); these are shown in Table 4. ADHD only has one set of factors influencing it: A1, C1, and E1. The variance of verbal ability is decomposed into two different sets of factors: those that are shared with $\mathrm{ADHD}$ (i.e., A1, C1, and E1) and those influencing verbal ability alone (i.e., A2, C2, and E2). CD is composed into three different sets of factors. The first set of factors is shared with both $\mathrm{ADHD}$ and verbal ability (i.e., A1, C1, and E1); 17\% of the variance of $\mathrm{CD}$ is due to genetic and environmental influences that are shared with ADHD and verbal ability. The second set of factors (i.e., A2, C2, and E2) is shared with verbal ability only; this set of factors contributes very little to the variance of CD (about $2 \%$ total). The third set of factors (i.e., A3, C3, and E3) is specific to $\mathrm{CD}$, and explains most of the variance of CD (i.e., $68 \%$ ).

\section{DISCUSSION}

The aim of the present study was to increase the understanding of the etiology of $\mathrm{CD}$, and assess whether low verbal ability shares unique variance with $\mathrm{CD}$, above and beyond that shared with ADHD. We examined the etiology of CD by dividing the variance of CD into variance shared with ADHD and verbal ability, and the 
Table 3 | Within- and cross-trait, cross-twin correlations.

\begin{tabular}{|c|c|c|c|c|c|c|c|}
\hline & MZF & DZF & MZM & DZM & DZO & MZ all & DZ all \\
\hline \multicolumn{8}{|c|}{ WITHIN-TRAIT, CROSS-TWIN } \\
\hline ADHD & $0.412^{* *}$ & $0.253^{*}$ & $0.317 * *$ & $0.175^{*}$ & $0.165^{*}$ & $0.368 * *$ & $0.192^{* *}$ \\
\hline Verbal & $0.798 * *$ & $0.496^{* *}$ & $0.710^{* *}$ & $0.650 * *$ & $0.531 * *$ & $0.758^{* *}$ & $0.544^{* *}$ \\
\hline$C D$ & $0.587^{* *}$ & $0.449 * *$ & $0.637^{* *}$ & $0.439 * *$ & $0.299 * *$ & $0.612^{* *}$ & $0.385^{* *}$ \\
\hline \multicolumn{8}{|c|}{ CROSS-TRAIT, CROSS-TWIN } \\
\hline CD-verbal & $-0.170 * *$ & $-0.192^{* *}$ & -0.017 & -0.009 & -0.058 & $-0.100 * *$ & $-0.092 * *$ \\
\hline
\end{tabular}

*Indicates a significant $p$ value of $<0.05 ; * *$ indicates a significant $p$ value of $<0.01$.

Table 4 | Percent variance of ADHD, verbal ability, and CD.

\begin{tabular}{|c|c|c|c|c|c|c|c|c|c|c|}
\hline & A1 & C1 & E1 & A2 & C2 & E2 & A3 & C3 & E3 & AGE \\
\hline ADHD & $0.34^{* *}$ & 0.00 & $0.63^{* *}$ & - & - & - & - & - & - & $0.03^{* *}$ \\
\hline Verbal & 0.03 & 0.06 & 0.00 & $0.41^{* *}$ & 0.27 & $0.22^{* *}$ & - & - & - & - \\
\hline
\end{tabular}

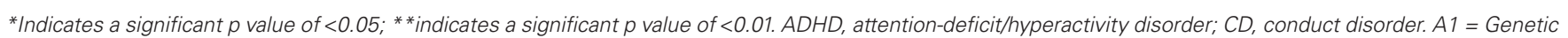

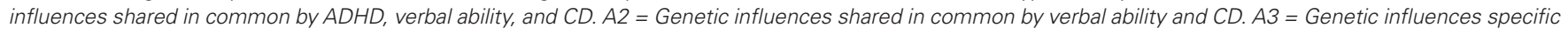

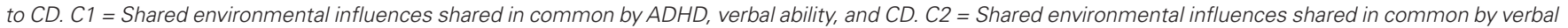

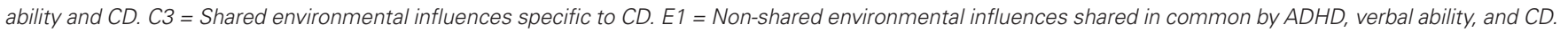
$E 2=$ Non-shared environmental influences shared in common by verbal ability and CD. E3 = Non-shared environmental influences specific to CD.

variance shared with verbal ability after controlling for ADHD. There was a small but statistically significant, negative correlation between verbal ability and CD and between verbal ability and ADHD. Dropping the genetic, shared environmental, and nonshared environmental influences on CD that are shared with verbal ability alone (after controlling for ADHD; i.e., dropping the paths $a_{32}, c_{32}$, and $e_{32}$ in Figure 1 ) did not lead to a significant decrement in the fit of the model. When the variance of CD was decomposed into influences that are shared in common with both verbal ability and ADHD, those shared in common only with verbal ability, and CD-specific influences, the influences shared in common only with verbal ability (i.e., A2, C2, and E2 in Figure 1) contributed very little to the variance of CD (about $2 \%$ of the total variance of CD). Overall, the results indicate that the common influences shared by ADHD, verbal ability, and CD have a significant effect on the variance of CD (approximately 16\%). Most of the variance of CD was not shared with verbal ability or ADHD (i.e., 68\%). In support of alternative hypothesis 2 , these results indicate that low verbal ability is not a significant risk factor for CD after controlling for ADHD.

These results suggest that the covariance between verbal ability and CD is significant, but that most of the covariance is associated with influences also shared in common with ADHD. Although one study (Déry et al., 1999) found that lower verbal skills characterize CD without ADHD, several researchers (e.g., Moffitt and Silva, 1988b; Henry and Moffitt, 1997; Hogan, 1999; Burke et al., 2002; Waschbusch, 2002) have suggested that the covariation between verbal skills and CD may be due to the significant comorbidity between ADHD and CD. The present study found little evidence of genetic and environmental influences on verbal ability associated with $\mathrm{CD}$ that are independent of those also influencing ADHD.
The association between verbal ability and ADHD/CD was smaller in the present study compared to those reported in previous studies. For example, Moffitt and Silva (1988b) compared the verbal IQ scores of controls and individuals with ADHD, delinquency, and both ADHD and delinquency; the standardized effect size $r$ for the relationship between verbal IQ and ADHD/delinquency in this sample ranged from 0.14 to 0.56 . Also, Elkins et al. (1997) noted that verbal IQ explained about 5\% of the variance of delinquency in 12- to 13-year-old participants in two longitudinal samples. In the present study, the correlation between verbal ability and $\mathrm{ADHD} / \mathrm{CD}$ was approximately -0.07 , indicating approximately $0.5 \%$ of variance explained. It is possible that there is a smaller association between verbal ability and ADHD/CD in the present study given our examination of the association between verbal ability and DSM-IV ADHD/CD (rather than a dimensional measure of delinquency or antisocial behavior; e.g., Elkins et al., 1997) in a population-based sample (i.e., rather than a clinic referred or delinquent sample; e.g., Moffitt and Silva, 1988b). Future directions include the incorporation of additional dimensional assessments of $\mathrm{ADHD}$ and $\mathrm{CD}$ symptoms from other sources (i.e., parents and teachers).

The results of the present study should be interpreted while considering the following limitations. The prevalence of psychiatric disorders such as $\mathrm{CD}$ and $\mathrm{ADHD}$ are low in the general population. Therefore, we did not constrain the diagnosis of CD and ADHD to follow additional DSM-IV criteria with respect to age of onset of symptoms or symptom clustering. However, we also examined subthreshold levels of CD and ADHD, and most of the statistical power in the analyses was from the subthreshold subjects. Given the small covariance between verbal ability and ADHD/CD, there 
was insufficient power to distinguish between the roles of genetic vs. environmental influences on the covariance. Another potential limitation was the adolescent self report of CD and ADHD assessed via the DISC. Compared to using multiple measures, basing the data solely on self reports may have decreased heritability estimates (Eaves et al., 1997). There is also some criticism regarding reliability of the DISC (e.g., McMahon and Frick, 2005; Silverman and Ollendick, 2005). However, in the present sample, we found significant twin correlations for both ADHD and CD in MZ and DZ twins, suggesting evidence of reliability and validity of the DISC in assessing $\mathrm{ADHD}$ and $\mathrm{CD}$. Another potential limitation was the use of the vocabulary subtest from the WISC-III and WAIS-III. Due to time constraints, this was the only verbal test administered. However, it is a reliable and valid measure, and is the subtest with the highest loading on the Verbal Comprehension factor in both instruments (Wechsler, 1997b).

To date, little research examining how verbal ability, ADHD, and CD are related has been conducted. The present study's results indicate that it may be helpful to consider etiological factors shared in common by all three traits when conducting research examining the covariance among them. Moreover, when examining the association between verbal ability and $\mathrm{CD}$, considering the comorbidity between ADHD and CD may help researchers understand the origins of verbal deficits in individuals with CD. In order to understand why verbal deficits are a risk factor for CD, it may be important to understand why verbal deficits are associated with ADHD. For example, attention problems in early childhood may be a moderator in the association between verbal ability and social relationships and school performance (e.g., Moffitt, 1993). Additionally, studies have examined the deficits common to both ADHD and reading disability, a disorder characterized by significant verbal ability deficits. For example, ADHD and reading disability are both characterized by deficits in processing speed (Willcutt et al., 2005, 2010), and genes influencing processing speed also increase the risk for ADHD and reading disability (Willcutt et al., 2010). Identification of such genetically influenced, common deficits may help explain the covariation among verbal ability, $\mathrm{ADHD}$, and CD.

\section{ACKNOWLEDGMENTS}

This research was supported by grants from the National Institutes of Health (HD010333, DA011015, HD050346, HD007289). The authors thank the participants and research assistants for their participation and assistance with this project.

\section{REFERENCES}

American Psychiatric Association. (1994). Diagnostic and Statistical Manual of Mental Disorders, 4th Edn. Washington, DC: American Psychiatric Association.

American Psychiatric Association. (2000). Diagnostic and Statistical Manual of Mental Disorders, 4th Edn (Text Revised). Washington, DC: American Psychiatric Association.

Angold, A., Costello, E. J., and Erkanli, A. (1999). Comorbidity. J. Child Psychol. Psychiatr. 40, 57-87.

Burke, J., Loeber, R., and Birmaher, B. (2002). Oppositional defiant disorder and conduct disorder: a review of the past 10 years, part II. J. Am. Acad. Child Adolesc. Psychiatry 41, 1275-1292.

Derks, E. M., Dolan, C. V., and Boomsma, D. I. (2004). Effects of censoring on parameter estimates and power in genetic modeling. Twin Res. 7, 659-669.

Déry, M., Toupin, J., Pauzé, R., Mercier, H., and Fortin, L. (1999). Neuropsychological characteristics of adolescents with conduct disorder: association with attention-deficit-hyperactivity and aggression. J. Abnorm. Child Psychol. 27, 225-236.

Dick, D. M., Viken, R. J., Kaprio, J., Pulkkinen, L., and Rose, R. J. (2005). Understanding the covariation among childhood externalizing symptoms: genetic and environmental influences on conduct disorder, attention deficit hyperactivity disorder, and oppositional defiant disorder symptoms. J. Abnorm. Child Psychol. 33, 219-229.

Eaves, L. J., Silberg, J. L., Meyer, J. M., Maes, H. H., Simonoff, E., Pickles, A., Rutter, M., Neale, M. C., Reynolds, C. A., Erikson, M. T., Heath, A. C., Loeber, R., Truett, K. R., and Hewitt, J. K. (1997). Genetics and developmental psychopathology: 2. The main effects of genes and environment on behavioral problems in the Virginia twin study of adolescent behavioral development. J. Child Psychol. Psychiatr. 38, 965-980.

Ehringer, M. A., Rhee, S. H., Young, S., Corley, R., and Hewitt, J. K. (2006). Genetic and environmental contributions to common psychopathologies of childhood and adolescence: a study of twins and their siblings. J. Abnorm. Child Psychol. 34, 1-17.

Elkins, I. J., Iacono, W. G., Doyle, A. E., and McGue, M. (1997). Characteristics associated with the persistence of antisocial behavior: results from recent longitudinal research. Aggress. Violent Behav. 2, 101-124.

Faraone, S. V., Perlis, R. H., Doyle, A. E., Moller,J.W., Goralnick, J.J., Holmgren, M. A., and Sklar, P. (2005). Molecular genetics of attention-deficit/hyperactivity disorder. Biol. Psychiatry 57, 1313-1323.

Farrington, D. P., and Hawkins, J. (1991). Predicting participation, early onset, and later persistence in officially recorded offending. Crim. Behav. Ment. Health 1, 1-33.

Henry, B., and Moffitt, T. (1997). "Neuropsychological and neuroimag- ing studies of juvenile delinquency and adult criminal behavior," in Handbook of Antisocial Behavior, eds D. Stoff and J. Breiling (New York, NY: Wiley), 280-288.

Hirschi, T., and Hindelang, M. (1977). Intelligence and delinquency: a revisionist review. Am. Sociol. Rev. 42, 571-587.

Hoekstra, R. A., Bartels, M., and Boomsma, D. I. (2007). Longitudinal genetic study of verbal and nonverbal IQ from early childhood to young adulthood. Learn. Individ. Differ. 17, 97-114.

Hogan, A. E. (1999). “Cognitive functioning in children with oppositional defiant disorder and conduct disorder," in Handbook of Disruptive Disorders, eds H.C. Quay and A.E.Hogan (New York, NY: Plenum Publishers), 317-335.

Hu, L., and Bentler, P. M. (1998). Fit indices in covariance structure modeling: sensitivity to under parameterized model misspecification. Psychol. Methods 3, 424-453.

Koenen, K. C., Caspi, A., Moffitt, T. E. Rijsdijk, F., and Taylor, A. (2006). Genetic influences on the overlap between low IQ and antisocial behavior in young children. J. Abnorm. Psychol. 115, 787-797.

Loney, B. R., Frick, P. J., Ellis, M., and Mc Coy, M. G. (1998). Intelligence, callous-unemotional traits, and antisocial behavior. J. Psychopathol. Behav. Assess. 20, 231-247.

Luria, A. R. (1963). "The role of speech in the formation of temporary con- nections and the regulation of behaviour in the normal and oligophrenic child," in Educational Psychology in the U.S.S.R, eds B. Simon and J. Simon (Stanford, CA: Stanford University Press), 83-97.

Lynam, D., Moffitt, T., and StouthamerLoeber, M. (1993). Explaining the relation between IQ and delinquency: class, race, test motivation, school failure, or self-control? J. Abnorm. Psychol. 102, 187-196.

McMahon, R. J., and Frick, P. J. (2005). Evidence-based assessment of conduct problems in children and adolescents. J. Clin. Child Adolesc. Psychol. 34, 477-505.

Moffitt, T. E. (1990a). Juvenile delinquency and attention deficit disorder: boys' developmental trajectories from age 3 to age 15. Child Dev. 61, 893-910.

Moffitt, T.E. (1990b). The neuropsychology of juvenile delinquency: a critical review. Crime Justice 12, 99-169.

Moffitt, T.E. (1993). Adolescence-limited and life-course persistent antisocial behavior: a developmental taxonomy. Psychol. Rev. 100, 674-701.

Moffitt, T. E., Lynam, D. R., and Silva, P.A. (1994). Neuropsychological tests predicting persistent male delinquency. Criminology 32, 277-300.

Moffitt, T. E., and Silva, P. A. (1988a). IQ and delinquency: a direct test of the difference detection hypothesis. J. Abnorm. Psychol. 97, 330-333.

Moffitt, T. E., and Silva, P. A. (1988b). Self-reported delinquency, 
neuropsychological deficit, and history of attention deficit disorder. $J$. Abnorm. Child Psychol. 16, 553-569.

Muthén, L. K., and Muthén, B. O. (19982004). Mplus User's Guide, 3rd Edn. Los Angeles, CA: Muthén \& Muthén.

Nadder, T. S., Rutter, M., Silberg, J. L., Maes, H. H., and Eaves, L. J. (2002). Genetic effects on the variation and covariation of attention deficithyperactivity disorder (ADHD) and oppositional-defiant disorder/ conduct disorder (Odd/CD) symptomatologies across informant and occasion of measurement. Psychol. Med. 32, 39-53.

Nigg, J. T., and Huang-Pollock, C. L. (2003). "An early-onset model of the role of executive functions and intelligence in conduct disorder/ delinquency," in Causes of Conduct Disorder and Juvenile Delinquency, eds B. B. Lahey, T. E. Moffitt, and A. Caspi (New York, NY: The Guilford Press), 227-253.

Petee, T. A., and Walsh, A. (1987). Violent delinquency, race, and the Wechsler performance-verbal discrepancy. $J$. Soc. Psychol. 127, 353-354.

Plomin, R., and Craig, I. (1997). Human behavioral genetics of cognitive abilities and disabilities. Bioessays 19, 1117-1124.

Plomin, R., Price, T. S., Eley, T. C., Dale, P. S., and Stevenson, J. (2002). Associations between behavior problems and verbal and nonverbal cognitive abilities and disabilities in early childhood. J. Child Psychol. Psychiatr. 43, 619-33.

Plomin, R., and Spinath, F. M. (2004). Intelligence: genetics, genes, and genomics. J. Pers. Soc. Psychol. 86, 112-129.

Quay, H. (1987). "Intelligence," in Handbook of Juvenile Delinquency, ed. H. Quay (New York, NY: Wiley), 106-117.

Rhea, S. A., Gross, A. A., Haberstick, B. C., and Corley, R. P. (2006). Colorado twin registry. Twin Res. Hum. Genet. 9, 941-949.

Rhee, S. H., and Waldman, I. D. (2002). Genetic and environmental influences on antisocial behavior: a meta-analysis of twin and adoption studies. Psychol. Bull. 128, 490-529.

Savitsky, J. C., and Czyzewski, D. (1978). The reaction of adolescent offenders and nonoffenders to nonverbal emotion displays. J. Abnorm. Child Psychol. 6, 89-96.

Shaffer, D., Fisher, P., Dulcan, M. K., Davies, M., Piacentini, J., SchwabStone, M. E., Lahey, B. B., Bourdon, K., Jensen, P. S., Bird, H. R., Canino, G., and Regier, D. A. (1996). The NIMH diagnostic interview schedule for children version 2.3 (DISC-2.3): description, acceptability, prevalence rates, and performance in the MECA study. Methods for the epidemiology of child and adolescent mental disorders study. J. Am. Acad. Child Adolesc. Psychiatry 35, 865-877.

Shaffer, D., Fisher, P., Lucas, C. P., Dulcan, M. K., and Schwab-Stone, M. E. (2000). NIMH diagnostic interview schedule for children version IV (NIMH DISC-IV): description, differences from previous versions, and reliability of some common diagnoses. J. Am. Acad. Child Adolesc. Psychiatry 39, 28-38.
Silverman, W. K., and Ollendick, T. H. (2005). Evidence-based assessment of anxiety and its disorders in children and adolescents. J. Clin. Child Adolesc. Psychol. 34, 380-411.

Stallings, M. C., Hewitt, J., Lessem, J. M., Young, S. E., Corley, R., Mikulich, S. K., and Crowley, T.J. (2001). Modeling the familial transmission of alcohol dependence symptom counts in clinical and control family pedigrees. Behav. Genet. 31, 470.

Thapar, A., Harrington, R., and McGuffin, P. (2001). Examining the comorbidity of ADHD-related behaviours and conduct problems using a twin study design. Br. J. Psychiatry 179, 224-229.

Waschbusch, D. A. (2002). A meta-analytic examination of comorbid hyperactive-impulsive-attention problems and conduct problems. Psychol. Bull. 128, 118-150.

Wechsler, D. (1944). The Measurement of Adult Intelligence, 3rd Edn. Baltimore, MD: The Williams \& Wilkins Company.

Wechsler, D. (1991). Wechsler Intelligence Scale for Children - III. San Antonio, TX: The Psychological Corporation.

Wechsler, D. (1997a). Wechsler Adult Intelligence Scale - III. San Antonio, TX: The Psychological Corporation.

Wechsler, D. (1997b). Wechsler Adult Intelligence Scale - III and Wechsler Memory Scale Technical Manual. San Antonio, TX: The Psychological Corporation.

Willcutt,E. G., Betjemann, R.S., McGrath, L. M., Chhabildas, N. A., Olson, R. K., DeFries, J. C., Pennington, B. F. (2010). Etiology and neuropsychol- ogy of comorbidity between RD and ADHD: the case for multiple-deficit models. Cortex 46, 1345-1361.

Willcutt, E. G., Pennington, B. F., Olson, R. K., Chhabildas, N., and Hulslander, J. (2005). Neuropsychological analyses of comorbidity between reading disability and attention deficit hyperactivity disorder: in search of the common deficit. Dev. Neuropsychol. 27, 35-78.

Wilson, J. Q., and Herrnstein, R. J. (1985). Crime and Human Nature. New York, NY: Simon \& Schuster.

Conflict of Interest Statement: The authors declare that the research was conducted in the absence of any commercial or financial relationships that could be construed as a potential conflict of interest.

Received: 31 July 2010; accepted: 16 February 2011; published online: 03 March 2011.

Citation: Smith AK, Stasi SM, Rhee SH, Corley RP, Young SE and Hewitt JK (2011) The role of attention-deficit/ hyperactivity disorder in the association between verbal ability and conduct disorder. Front. Psychiatry 2:3. doi: 10.3389/ fpsyt.2011.00003

This article was submitted to Frontiers in Child and Neurodevelopmental Psychiatry, a specialty of Frontiers in Psychiatry. Copyright $\odot 2011$ Smith, Stasi, Rhee, Corley, Young and Hewitt. This is an open-access article subject to an exclusive license agreement between the authors and Frontiers Media SA, which permits unrestricted use, distribution, and reproduction in any medium, provided the original authors and source are credited. 